\title{
Editorial: Is macroprolactinemia just a diagnostic pitfall?
}

\author{
Marcello D. Bronstein
}

Published online: 29 January 2012

(C) Springer Science+Business Media, LLC 2012

As hyperprolactinemia is involved in the impairment of the gonadotropic axis, serum prolactin (PRL) assessment is often required in the clinical scenario of menstrual disturbances, infertility, loss of libido, galactorrhea, osteoporosis, and bone fractures [1-3]. Nevertheless, the excessive requirement of PRL determination led to an important laboratory pitfall: the find of hyperprolactinemia in subjects with few or no symptoms related to such condition.

Human PRL in circulation has marked size heterogeneity, mainly with three forms: $23 \mathrm{kDa}$ (monomeric or little PRL), $50 \mathrm{kDa}$ (dimeric or big PRL), and $150-170 \mathrm{kDa}$ (big-big prolactin or macroprolactin, the three forms being indistinguishable by routine assays [4]. Nevertheless, monomeric PRL, the most common form, is considered to be the biological active one, whereas molecular aggregates as macroprolactin are regarded to have low biologic activity. Therefore, the predominance of macroprolactin, associated with normal levels of monomeric PRL, may lead to the laboratorial diagnosis of hyperprolactinemia in subjects with no symptoms, ending up in unnecessary treatment approaches. The gel-filtration chromatography is the goldstandard method to confirm the presence of big-big PRL, but is a costly and time-consuming process. For routine purposes, precipitation with polyethylene glycol (PEG) is an excellent screening method [5]. The predominant molecular form recovered (i.e., assayed after precipitation) is the highly biologically active monomeric PRL. Usually considering macroprolactin $>60 \%$ of total PRL, macroprolactinemia has been shown as a common finding,

\section{D. Bronstein $(\bowtie)$}

Neuroendocrine Unit, Division of Endocrinology and Metabolism, Hospital das Clinicas, University of Sao Paulo, Sao Paulo, SP, Brazil

e-mail: mdbronstein@uol.com.br occurring in $8-42 \%$ of all cases of hyperprolactinemia. The reason for such large range can be attributed both to selection bias and the use of diverse assays, which differently recognize the amount of big-big PRL in each sample [6].

Macroprolactin biologic activity is still controversial in the literature. Studies in vitro with rat $\mathrm{Nb} 2$ cell bioassays show either its presence or absence. To explain the presence of activity in vitro but not in vivo, it was hypothesized that its high molecular weight impairs capillary barrier crossing and its action on target cells. Moreover, the $\mathrm{Nb} 2$ cells are a PRL-dependent rat immune cell line expressing a mutant form of PRL receptor, so a bioassay using cells harboring human PRL receptors which addresses the biologic activity of macroprolactin should be more reliable. In fact, a study by Glezer et al. [7] showed that sera of individuals with macroprolactinemia presented lower biological activity in a bioassay using a mouse cell transfected with the long form of human PRL receptor as compared to the rat $\mathrm{Nb} 2$ bioassay. Moreover, Hattori et al. [8] assume that the bioactivity of macroprolactin in the $\mathrm{Nb} 2$ bioassay is due to dissociation of monomeric PRL from the autoantibodies as a result of the longer incubation than in a bioassay using human breast cancer cells. Despite these controversies in the literature concerning the biologic activity of PRL aggregates, most patients with macroprolactinemia do not manifest clinical features related to hyperprolactinemia.

This Endocrine issue brings two publications from Turkey addressing the macroprolactin matter. In one study, Isik et al. [9] enrolled 337 hyperprolactinemic individuals, and found that in $26.1 \%$ of them macroprolactinemia prevailed, based on a percentage of PRL in the serum supernatant $<40 \%$ after PEG precipitation. Subjects with PRL recover value of $\geq 40 \%$ were considered with absence of 
macroprolactin and recorded as harboring monomeric hyperprolactinemia. A comparison of clinical presentations did not reveal a significant difference between the macroprolactin and monoPRL groups concerning the rates of amenorrhea, irregular menses, erectile dysfunction, infertility, gynecomastia, and headaches. Galactorrhea was encountered more frequently in women from the monoPRL group compared to the macroprolactin group. Moreover, more women in the macroprolactin group were asymptomatic compared to those in the monoPRL group. In addition, abnormal MRI findings were more prevalent in the monoPRL group. The authors concluded that macroprolactinemia should be considered a pathological biochemical variant of hyperprolactinemia that may present with any of the conventional symptoms and radiological findings generally associated with elevated PRL levels. They believe that discriminating monomeric PRL and macroprolactin is clinically irrelevant and therefore the treatment of a patient referred for hyperprolactinemia should be based on the presence or absence of symptoms, regardless the monomeric PRL or macroprolactin levels.

Although agreeing that treatment should be based on clinical symptoms, I must point out some caveats regarding the statement that macroprolactinemia is a pathological condition: (1) Selection bias: The serum PRL assay was performed only in those individuals with hyperprolactinemia-related symptoms or also asymptomatic subjects were assessed in "routine" laboratory work-up? (2) Macroprolactin quantification varies amongst different assays [6]. (3) The presence of macroprolactin in $>60 \%$ of total serum PRL do not necessarily rules-out supranormal levels of monomeric PRL [6]. (4) Galactorrhea and symptomatic patients were more prevalent, as well as a trend to the presence of amenorrhea, in the monomeric PRL group.

In the other article, Tamer et al. [10] focused on pituitary imaging of 161 patients with hyperprolactinemia, $37.26 \%$ with macroprolactinemia. Pituitary adenomas were identified in the MRIs of 16 (26.7\%) macroprolactinemic patients and of $56(55.4 \%)$ patients with true hyperprolactinemia. Although, showing that pituitary adenomas were less common in patients with macroprolactinemia compared to patients with true hyperprolactinemia (all of them, except one with a $12 \mathrm{~mm}$ macroadenoma, harbored microadenomas) they stated that the prevalence of pituitary adenomas may be higher than it is presumed. Concerning this statement, I must point out that the presence of 1.5 Tesla MRI images suggestive of microadenomas in the general population can reach $10 \%$ of cases [11], and probably this percentage will increase with more accurate MRI devices. This is due both to incidental asymptomatic tiny tumors which can reach $26.7 \%$ of autopsies in the general population or imaging artifacts due to anatomic variants of the septation within the sphenoid sinus. Therefore, in the presence of hyperprolactinemia in a macroprolactinemic asymptomatic subject, the MRI evaluation can lead to a double diagnostic pitfall [12].

We should therefore be aware that the presence of macroprolactinemia do not rule-out clinical conditions linked to hyperprolactinemia: in patients with prolactinoma or other causes of pathological hyperprolactinemia and even in drug-induced hyperprolactinemia, macroprolactin may co-exists with elevated levels of the biological active, monomeric PRL, without meaning that macroprolactin has intrinsic biological activity. Therefore, I would suggest the avoidance of routine serum PRL assessment. In addition, to avoid unnecessary image evaluation as well as medical or surgical procedures, macroprolactin screening is mandatory when clinical features and serum PRL assay results are conflicting.

\section{References}

1. M.D. Bronstein, Disorders of prolactin secretion and prolactinomas, in Endocrinology, 6th edn., ed. by J.L. Jameson, L.J. De Groot (Saunders, Philadelphia, 2010), pp. 333-357

2. F.F. Casanueva et al., Guidelines of the pituitary society for the diagnosis and treatment of prolactinomas. Clin. Endocrinol. 65, 265-273 (2006)

3. G. Mazziotti et al., Vertebral fractures in males with prolactinomas. Endocrine 39, 288-293 (2011)

4. Y.N. Sinha, Structural variants of prolactin: occurrence and physiological significance. Endocr. Rev. 16, 354-369 (1995)

5. J.G. Vieira et al., Extensive experience and validation of polyethylene glycol precipitation as a screening method for macroprolactinemia. Clin. Chem. 44, 1758-1759 (1998)

6. T.P. Smith et al., Gross variability in the detection of prolactin in sera containing big big prolactin (macroprolactin) by commercial immunoassays. J. Clin. Endocrinol. Metab. 87, 5410-5415 (2002)

7. A. Glezer et al., Human macroprolactin displays low biological activity via its homologous receptor in a new sensitive bioassay. J. Clin. Endocrinol. Metab. 91, 1048-1055 (2006)

8. N. Hattori et al., Anti-prolactin (PRL) autoantibodies suppress PRL bioactivity in patients with macroprolactinaemia. Clin. Endocrinol. 68, 72-76 (2008)

9. Isik $\mathrm{S}$, et al., Clinical and radiological findings in macroprolactinemia. Endocrine (2012). doi:10.1007/s12020-011-9576-9

10. Tamer G, et al., Prevalence of pituitary adenomas in macroprolactinemic patients may be higher than it is presumed. Endocrine 41(1), 138-143 (2012)

11. W.A. Hall et al., Pituitary magnetic resonance imaging in normal human volunteers: occult adenomas in the general population. Ann. Intern. Med. 120, 817-820 (1994)

12. A. Glezer et al., Pitfalls in pituitary diagnosis: peculiarities of three cases. Clin. Endocrinol. 57, 135-139 (2002) 